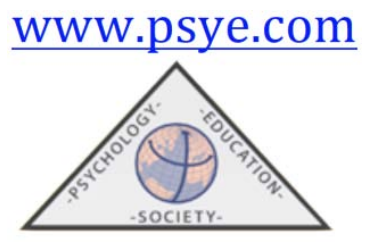

(C) Psychology, Society, \& Education, 2017. Vol. 9(1), pp. 105-119

ISSN 2171-2085 (print) / ISSN 1989-709X (online)

\title{
Análisis de las relaciones entre creencias epistemológicas sobre la matemática y rendimiento académico
}

\author{
Annia Esther VIZCAINO ESCOBAR* y Mayra MANZANO MIER** \\ (*) Universidad Central “Marta Abreu” de Las Villas, Cuba (**) Universidad de La Habana, Cuba
}

(Recibido 16 Mayo, 2016; Aceptado 25 Junio, 2016)

RESUMEN: La investigación toma como referencia el Paradigma Multidimensional propuesto por Marlene Schommer y colaboradores, para determinar las dimensiones de creencias epistemológicas sobre la Matemática que se relacionan con el rendimiento académico de esta asignatura. Participaron 522 alumnos de secundaria básica de la provincia de Villa Clara. Se constató que los estudiantes poseen creencias poco productivas para el aprendizaje de esta ciencia afectando los resultados docentes, así por ejemplo: cuando creen en la simplicidad del conocimiento matemático, en la presencia de una autoridad como fuente en la cual radica la información, que no son capaces de aprenderla de manera autodeterminada; que el aprendizaje de la Matemática no requiere del esfuerzo personal, sino que es una habilidad que se posee al nacer peor es su rendimiento. Las correlaciones positivas entre el rendimiento y la dimensión Conocimiento relativo indican que cuando los alumnos creen que el conocimiento matemático es relativo obtienen mejores resultados.

Palabras clave: epistemología personal, aprendizaje, alumnos, secundaria básica

\section{Analysis of relations between epistemological beliefs about mathematics and academic performance}

\begin{abstract}
The research draws on the Multidimensional Paradigm proposed by Marlene Schommer et al, to determine the dimensions of epistemological beliefs about mathematics that relate to the achievement of this subject. 522 students attended junior high school in the province of Villa Clara. It was found that students have little productive beliefs for learning this science affecting learning outcomes, so for example, when they believe in the simplicity of mathematical knowledge, in the presence of an authority as a source in which lies the information not they are able to learn selfdetermined way; learning of mathematics does not require personal effort, but it is a skill that has at birth worse performance. Positive correlations between performance and relative dimension Knowledge indicate that when students believe that mathematical knowledge is relative get better results.
\end{abstract}

Keywords: personal epistemology, learning, students, junior high.

Correspondencia: Annia Esther Vizcaino Escobar, e-mail: annia@uclv.edu.cu. Carretera a Camajuaní \# 258 / Tomás Ruíz y Ana Pegudo. Santa Clara, Villa Clara, Cuba. 
El proceso enseñanza-aprendizaje de la Matemática constituye uno de los ámbitos de la investigación científica que mayor interés ha despertado en los últimos años. Diversas evaluaciones a nivel internacional sobre rendimiento en la Matemática (INECSE, 2010; MEC, 2013; OCDE, 2010; PISA, 2012) revelan que un alto porcentaje de alumnos y alumnas fracasan en la educación secundaria y muestran dificultades para superar con éxito esta materia.

Distintas teorías y autores (Abrate, Pochulu, \& Vargas, 2006; Balbi, 2010; Campanario \& Otero, 2000; Carrillo, 2009; Coll, Palacios, \& Marchesi, 2008; Chevallard, Bosch, \& Gascón, 1997; Edwards, Esmonde, \& Wagner, 2011; Mason, 2003) investigan las causas. Schunk y Ertmer (2000) se lo atañen a la autoeficacia percibida para aprender esta ciencia; Miñano y Castejón (2011); Rosário et al. (2012) así como Zimmerman y Schunk (2011) a las estrategias de aprendizaje utilizadas. Por su parte Plant, Ericsson, Hill, y Asberg (2005) consideran que el tiempo dedicado al estudio de esta materia constituye un factor determinante en la obtención de sus resultados. Podrían mencionarse otras variables como el nivel educativo de los padres (Davis-Kean, 2005) o las que tienen que ver con la competencia del profesor de Matemática, su dominio de la ciencia que imparte y de los saberes didácticos y pedagógicos asociados a esta asignatura (Barberá \& Gómez, 1996; Font, 2011). Se señalan además, las características del conocimiento matemático (Bishop, 1999).

La mayoría de las investigaciones que han abordado la problemática se centran en las deficiencias o dificultades que pueden tener alumnos y alumnas, los profesores, el currículo, su implementación, los cánones que impone la sociedad de hoy. En menor cuantía se encuentran trabajos que se orienten a explorar las formas en que docentes y estudiantes se aproximan al conocimiento matemático desde una perspectiva personal y como ello facilita o no obtener mejores resultados.

Una alternativa que ha sido considerada recientemente, es el estudio de las creencias sobre la naturaleza y origen del conocimiento y del aprendizaje o creencias epistemológicas, constructo que ha sido retomado en esta investigación como una de las causas de la ejecución académica en Matemática. Interés que está marcado por potenciar el rol activo de alumnos y alumnas en el proceso de aprendizaje, reconociendo la implicación personal en la construcción de significados y la influencia del contexto social.

A pesar de la diversidad de términos empleados parece existir cierto acuerdo en que:

Las creencias epistemológicas contienen las suposiciones básicas de las personas en relación al conocimiento y el aprendizaje, a sus límites, a la firmeza que puede tener un determinado sistema de conocimientos y cómo funcionan en general el aprendizaje y la adquisición del conocimiento. (Hofer, 2008, p. 117).

Este supuesto resalta el rol activo del sujeto en su relación con el conocimiento. En el desarrollo de los procesos que realiza para conocer va configurando una forma de aprehender el conocimiento, la que a su vez se convierte en un estado de disposición previo, que origina nuevos aprendizajes (Leal, 2011).

Desde este referente, consideramos que las creencias ofrecen una ayuda ventajosa para comprender los resultados del aprendizaje, en tanto integran las posturas personales en relación a la naturaleza del conocimiento y las formas de adquirirlo.

En el marco de la línea metacognitiva se identifican los trabajos de Marlene Schommer. 
Schommer (1994) realiza un intento de resolver los resultados contradictorios que hasta ese momento se habían encontrado en la investigación de las creencias epistemológicas. Así, las presenta como:

Un constructo multidimensional constituido por un sistema de creencias que posee el individuo acerca de la naturaleza del conocimiento y el aprendizaje, las cuales son relativamente independientes entre sí. Es un sistema porque hay más de una creencia, se puede considerar más de una dimensión de las mismas y es más o menos independiente porque una persona puede tener al mismo tiempo unas creencias a un nivel sofisticado y otras a un nivel muy simple, poseen un desarrollo asincrónico. (Schommer, 1990, p. 499).

Presenta inicialmente cinco dimensiones, las cuales hacen referencia a lo siguiente:

La certeza del conocimiento: se corresponde con el nivel de relatividad que se le puede adjudicar a un conocimiento o idea según la claridad de las respuestas obtenidas, entendiéndolo como algo absoluto o provisional.

La estructura del conocimiento: esta dimensión está relacionada con la organización del conocimiento, si la creencia se acerca más a concebirlo como parcelas aisladas, desagregadas o como sistema de conceptos altamente entrelazados, interrelacionados.

El control de su adquisición: esta dimensión abarca desde considerar que la habilidad para aprender es fija, establecida desde el nacimiento o por factores de tipo genético, hasta la consideración de que el aprendizaje puede ser modificado y desarrollado de acuerdo a las experiencias en los diferentes contextos.

La velocidad de su adquisición: abarca la creencia acerca del tiempo que toma a una persona aprender o comprender algo. Se contraponen procesos de adquisición rápida con procesos de construcción lenta del conocimiento, es decir, si el aprendizaje ocurre de forma rápida, inmediata, del tipo todo o nada o su adquisición es gradual.

El origen o fuente del conocimiento: se refiere a la procedencia del conocimiento, al grado de confiabilidad que posee una información según sea su origen, visto como algo impuesto externamente y proporcionado por la autoridad, hasta derivado de la razón o del descubrimiento personal.

El estudio de las creencias desde el Paradigma Multidimensional constituye el supuesto básico y general del cual se parte para esta investigación, ofrece una alternativa objetiva y coherente para una mayor aproximación a la influencia que ellas ejercen en la resolución de problemas y la acción.

En el ámbito de las creencias epistemológicas sobre la Matemática se encuentran los trabajos de Schommer y Duell (2013) quienes consideraron que si los alumnos creen que la matemática no es útil en su vida o en una carrera futura, no querrán dedicar el tiempo y el esfuerzo necesario para aprenderla; al mismo tiempo, en esos estudios mostraron que las creencias epistemológicas sobre la Matemática guardan relación con el uso eficaz de estrategias de solución y con el logro académico.

También Schoenfeld (1989), en su interés por indagar sobre este tema, realizó una investigación con estudiantes de Secundaria Básica, a partir de la cual, después de haberlos visto resolver problemas geométricos y estimularlos a que hablaran sobre ello, descubrió que muchos alumnos creen que los matemáticos nacen con la habilidad para la Matemática y que 
los problemas de este tipo no deben demorar más de 12 minutos en resolverse. De esta manera, en opinión de Schommer, Schoenfeld es el precursor de la creencia sobre la habilidad de aprender y el precursor de la creencia en la velocidad del aprendizaje (Schommer, 2004).

Otros autores como Kloosterman y Stage (1992); Kloosterman y Cougan (1994); Muis (2004) refieren que las creencias epistemológicas acerca de la Matemática con influencia negativa en la motivación y en la comprensión de los estudiantes son consideradas poco ventajosas para el aprendizaje.

El análisis del vínculo entre las creencias y el rendimiento académico ha sido objeto de numerosos autores, encontrándose distintas relaciones de influencia. Schommer, Crouse, y Rhoder (1992) por ejemplo, identificaron que las estrategias de aprendizaje actúan como mediadoras de los efectos de las creencias epistemológicas sobre el rendimiento académico.

Cano (2005)comprobó que la influencia de las creencias epistemológicas en el rendimiento académico es directa e indirecta a través de los enfoques de aprendizaje.

Coincidiendo con los criterios de Vila y Callejo (2004), las creencias matemáticas de un individuo, su punto de vista matemático, forman parte de un sistema regulador de su estructura de conocimiento, dentro de este marco actúa y piensa y a su vez este marco influye fuertemente en su rendimiento. Entendidas así, las creencias sobre la matemática, se conciben como trama de fondo de sus motivaciones, sus experiencias, sus conocimientos, necesidades y ejercen influencia sustantiva en las prácticas de esta materia (Steiner, 2007; Walker, 2007).

Teniendo como referentes los presupuestos anteriores esta investigación plantea como problema científico ¿cómo se relacionan las dimensiones de creencias sobre la Matemática con los niveles de rendimiento académico en que se encuentran los alumnos y alumnas de secundaria básica? El objetivo general que guía el proceso investigativo está orientado a determinar las relaciones que se establecen entre las dimensiones de creencias sobre la Matemática y los niveles de rendimiento académico en esta materia que alcanzan alumnos y alumnas de secundaria básica, cuestión aún controvertida en el campo investigativo. Como objetivos específicos se definieron: Identificar los niveles de rendimiento académico en que se encuentra los alumnos y las alumnas de secundaria básica; Caracterizar los grupos de rendimiento académico encontrados y Explorar las dimensiones de creencias sobre la Matemática que se relacionan y que mejor predicen el rendimiento académico en esta asignatura.

\section{Método}

\section{Participantes}

Fueron escogidos 522 estudiantes de Villa Clara, en la Tabla 1 se pueden observar las variables sociodemográficas que caracterizan la muestra. 
Tabla 1. Distribución de las Variables Sociodemográficas de los Participantes

\begin{tabular}{llll}
\hline & & Participantes & \% \\
\hline \multirow{4}{*}{ Grados } & Séptimo & 155 & 29.7 \\
& Octavo & 174 & 33.3 \\
& Noveno & 193 & 37.0 \\
& Total & 522 & 100 \\
\hline \multirow{4}{*}{ Edad } & 12 & 111 & 21.3 \\
& 13 & 180 & 34.5 \\
& 14 & 197 & 37.7 \\
& 15 & 34 & 6.5 \\
& Total & 522 & 100 \\
\hline \multirow{3}{*}{ Sexo } & Femenino & 254 & 48.7 \\
& Masculino & 268 & 51.3 \\
& Total & 522 & 100 \\
\hline
\end{tabular}

\section{Instrumento}

El Cuestionario de creencias epistemológicas sobre la Matemática para estudiantes de secundaria básica utilizado en el presente estudio es la versión validada por Vizcaino, Manzano, y Casas (2015).

Dicho cuestionario está integrado por 18 ítems que evalúan cuatro dimensiones de creencias sobre la Matemática: Habilidad para aprender la Matemática (ítems 1, 11, 8, 3 y 15); Estructura y Fuente del conocimiento que se integran en una sola dimensión (ítems 7, 2, 4, 12, 17, 6); Estabilidad (Certeza) del conocimiento (ítems 10, 14, 5, 16); Velocidad en la adquisición y Aplicabilidad de la Matemática, que también se integran en una dimensión (ítems 9,18 y 13). Se califica en una escala que presenta valores del 1 al 7 , donde 1 es totalmente en desacuerdo, 4 corresponde al valor neutral y 7 totalmente de acuerdo.

\section{Procedimiento}

Se adoptó como medida del rendimiento académico el promedio de calificaciones obtenido por los alumnos y las alumnas en el curso escolar 2014-2015. Se tomaron del registro docente de Matemática, todas las evaluaciones sistemáticas y resultados de la prueba parcial, correspondientes a cada uno de los participantes en el estudio. Con la información obtenida se realizó un análisis de la media de sus aprendizajes, el valor más alto que podían obtener era 50 puntos.

Se conformó la escala de transformación a partir de los criterios de especialistas del Ministerio de Educación. Se utilizó la técnica de clasificación conglomerados en dos fases para la conformación de los grupos de acuerdo a los niveles del rendimiento académico según la escala. Para describir la composición de los grupos por niveles de rendimiento en Matemática se realizaron análisis de la media, la mediana, la varianza y la desviación típica. Se identificaron además las diferencias entre los conglomerados a partir del sexo y el grado.

Para la recogida de la información se utilizó el registro docente de la asignatura, documento oficial que refleja las calificaciones obtenidas por alumnos y alumnas según el 
sistema de evaluación que se aplica.

Como método para la obtención de resultados en cuanto a la definición de los niveles de rendimiento académico se concibió como escala: Nivel 1(Bajo): Estudiante con dificultades, calificaciones promedio por debajo de 40 puntos; Nivel 2 (Medio): Estudiante promedio, calificaciones promedio entre 40 y 47 puntos; Nivel 3 (Alto): Estudiante aventajado o de concurso, calificaciones promedio $>=47$.

Una vez identificados los niveles de rendimiento académico en que se encuentran alumnos y alumnas se determinó su relación con las dimensiones de creencias sobre la Matemática. Se utilizó el test de correlación Tau_b de Kendall. Para profundizar en las relaciones y corroborar los resultados se aplicó el test de Kruskal Wallis.

Se estimaron además las dimensiones de creencias que mejor predicen el rendimiento académico, realizando para ello un análisis de regresión.

Se aceptó la relación entre rendimiento académico en Matemática y creencias sobre esta ciencia cuando el valor de la significación del coeficiente de correlación de Tau_b de Kendall y el test de Kruskal Wallis fue $\mathrm{p}<0.05$. De igual forma se aceptó el valor predictivo de las creencias sobre el rendimiento en Matemática cuando el valor de la significación del ANOVA aplicado con la regresión fue de $\mathrm{p}<0.05$.

Se solicitó la disposición y consentimiento para colaborar en el estudio a los directivos, profesores y estudiantes de secundaria básica de la provincia de Villa Clara en la Región Central de Cuba. Se obtuvo la aprobación en todos los casos. En el caso de los alumnos y las alumnas se solicitó además el consentimiento a sus padres, los cuales estuvieron de acuerdo con que sus hijos participaran en la investigación. La escala se aplicó en las aulas de cada una de las instituciones escolares por psicólogos entrenados para la aplicación de pruebas colectivas a adolescentes. Los cuales respondieron al cuestionario de forma individual en un tiempo máximo de 20 minutos.

\section{Resultados}

Se obtuvieron 3 conglomerados que mostraron diferencias estadísticamente significativas según características del sistema de creencias sobre la matemática y el nivel de rendimiento en que se encuentran los estudiantes.

Como se observa en la Tabla 2, la mayor cantidad de alumnos y alumnas de secundaria básica objeto de estudio se encuentran en el nivel bajo (el $48.5 \%$ ) y son fundamentalmente del conglomerado 1; el nivel de aprendizaje medio está representado por alumnos y alumnas de los tres conglomerados, aunque predominan los de los grupos 2 y $3\left(8^{\mathrm{vo}}\right.$ y $\left.9^{\mathrm{no}}\right)$ y el nivel alto, está compuesto en su mayoría por los del grupo 2 , que son esencialmente de $8^{\text {vo }}$, nótese que a este nivel pertenecen pocos estudiantes. 
Tabla 2. Nivel de Rendimiento Académico en Matemática Según Grupos de Alumnos

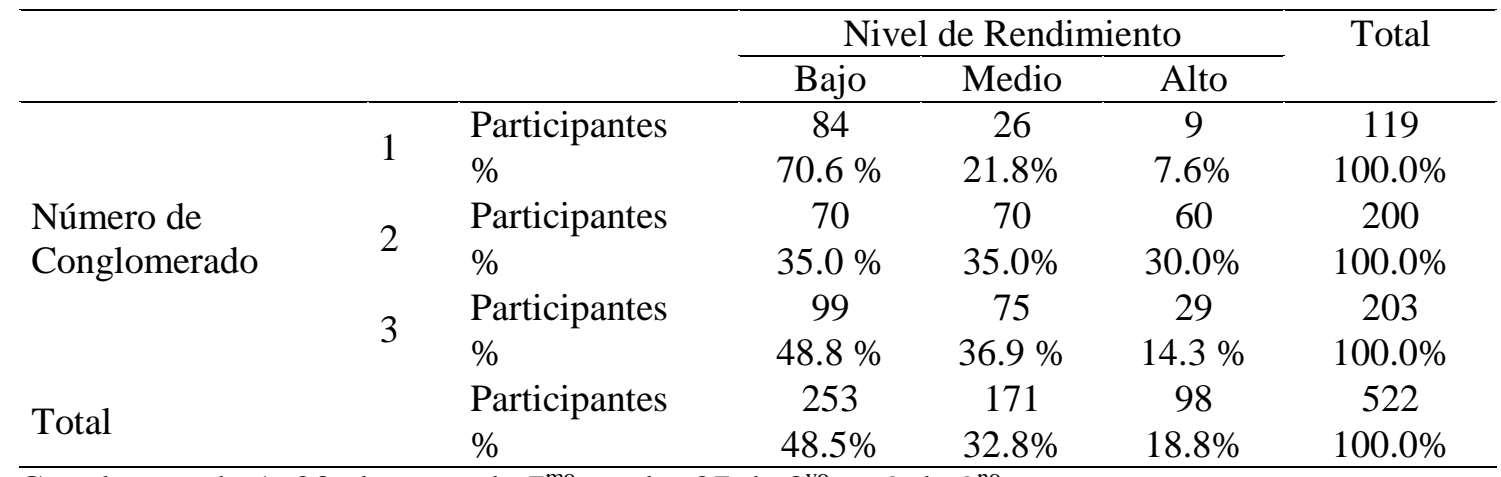

Conglomerado 1: 23 alumnos de $7^{\text {mo }}$ grado; 27 de $8^{\text {vo }}$ y 69 de $9^{\text {no }}$

Conglomerado 2: 70 alumnos de $7^{\text {mo }}$ grado; 78 de $8^{\text {vo }}$ y 52 de $9^{\text {no }}$

Conglomerado 3: 62 alumnos de $7^{\text {mo }}$ grado; 69 de $8^{\text {vo }}$ y 72 de $9^{\text {no }}$

En cuanto al sexo también se encontraron diferencias estadísticamente significativas. Como muestra la Figura 1, la mayor cantidad de estudiantes del nivel académico bajo pertenecen al sexo masculino (el $76 \%$ ), en los niveles medio y alto se encuentran con mayor frecuencia las féminas. Podría ser este un punto de continuidad de las investigaciones orientadas a las diferencias de género, en este trabajo se demuestra que cuando las creencias poseen menor desarrollo se obtiene menor calificación y eso se manifiesta con mayor frecuencia en los alumnos del sexo masculino.

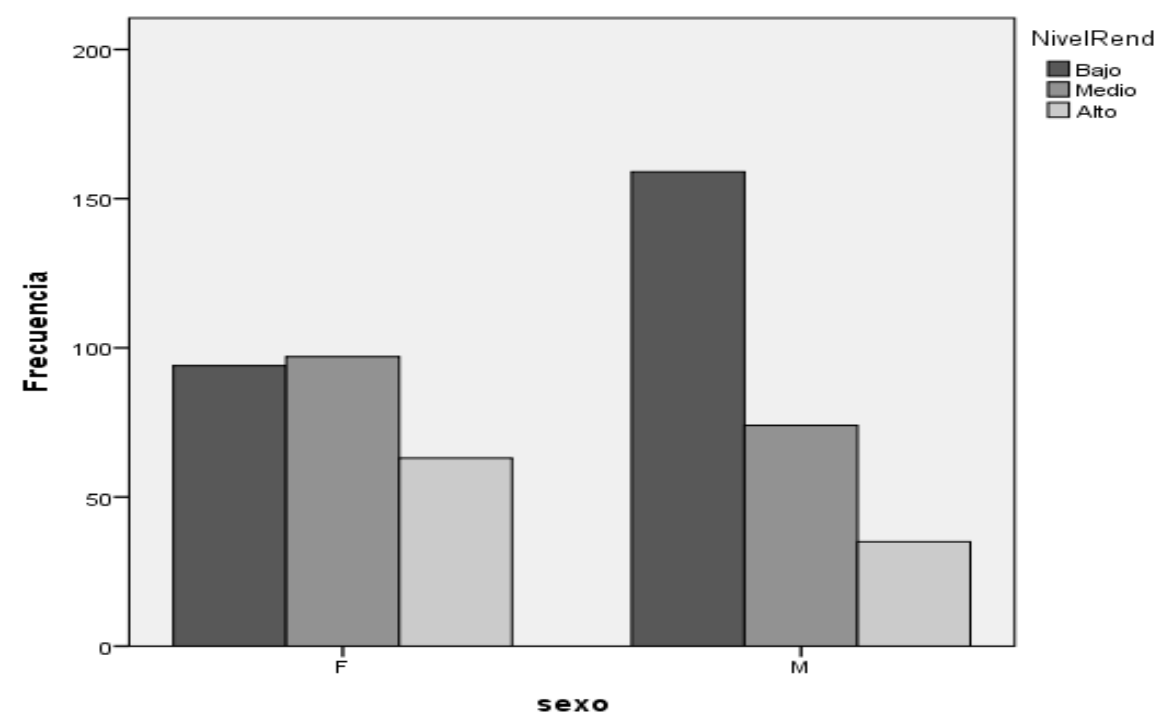

Figura 1. Niveles de rendimiento académico en matemática según el sexo. 


\section{Relaciones entre las dimensiones de creencias y el rendimiento académico}

Aunque en la literatura relacionada con el tema, no siempre se reflejan las relaciones de las creencias con el rendimiento, en algunas se encontraron resultados significativos, por ejemplo, en el estudio de Rodríguez (2005, p. 179) el factor "La habilidad para aprender es innata”, contribuyó con un coeficiente negativo a la ecuación de regresión, de forma tal que mientras más creían los estudiantes que la habilidad para aprender es innata, peor era su rendimiento académico. En el contexto cubano se encuentran la investigaciones de De la Yera (2013); Mederos (2013); Mendoza (2012); Morell (2011); Reynó de los Ríos (2014); Rodríguez (2013), quienes encontraron que a medida que los estudiantes disminuyen su creencia en que el conocimiento radica en la figura de la autoridad aumentan la calidad de su rendimiento académico.

El análisis de las relaciones entre las dimensiones de creencias y el rendimiento en matemática, realizado en este estudio a través del test de correlación Tau_b de Kendall, indicó correlaciones altamente significativas, no obstante, esta correlaciones no siempre son positivas, así por ejemplo, mientras los estudiantes creen que el aprendizaje de la Matemática no requiere del esfuerzo personal, sino que es una habilidad que se posee al nacer y de ello depende los resultados que obtenga peor es su rendimiento (Tabla 3). La Tabla 3 ilustra además que obtienen rendimiento bajo en Matemática cuando creen en la simplicidad de este conocimiento; en la presencia de una autoridad como fuente en la cual radica la información y que no son capaces de aprender de manera autodeterminada, porque requieren de una habilidad innata y porque deben aprenderlo todo de una vez.

Por otra parte, las correlaciones positivas entre el rendimiento y la dimensión Conocimiento relativo indican que cuando creen que el conocimiento matemático es relativo obtienen mejores resultados.

Si se observan el resto de las correlaciones encontradas las dimensiones del sistema de creencias que reflejan menor desarrollo o ingenuidad (Habilidad innata; Conocimiento simple, dependiente de la autoridad; Aprendizaje rápido e inaplicable), tienen una relación positiva, en la medida que aumenta una aumenta la otra, no se comportan de igual manera con la dimensión Conocimiento relativo que siempre establecen una relación negativa.

Tabla 3. Correlaciones entre el Sistema de Creencias sobre la Matemática y el Rendimiento Académico

\begin{tabular}{cccccc}
\hline $\begin{array}{c}\text { Variables de } \\
\text { relación }\end{array}$ & Rendimiento & $\begin{array}{c}\text { Habilidad } \\
\text { Innata }\end{array}$ & $\begin{array}{c}\text { Conocimiento } \\
\text { Simple } \\
\text { Autoridad }\end{array}$ & $\begin{array}{c}\text { Conocimiento } \\
\text { Relativo }\end{array}$ & $\begin{array}{c}\text { Aprendizaje } \\
\text { Rápido e } \\
\text { Inaplicable }\end{array}$ \\
\hline Rendimiento & 1,000 &,$- 122\left(^{* *}\right)$ &,$- 197\left(^{* *}\right)$ &, $147\left(^{* *}\right)$ &,$- 240\left(^{* *}\right)$ \\
$\begin{array}{c}\text { Habilidad } \\
\text { Innata }\end{array}$ &,$- 122\left(^{* *}\right)$ & 1,000 &, $231\left(^{* *}\right)$ &,$- 063\left(^{*}\right)$ &, $206\left(^{* *}\right)$ \\
$\begin{array}{c}\text { Conocimiento } \\
\text { Simple }\end{array}$ &,$- 197\left(^{* *}\right)$ &, $231\left(^{* *}\right)$ & 1,000 &,$- 063\left(^{*}\right)$ &, $279\left(^{* *}\right)$ \\
Autoridad & & & & &
\end{tabular}




\begin{tabular}{lccccc}
$\begin{array}{l}\text { Conocimiento } \\
\text { Relativo }\end{array}$ &, $147(* *)$ &,$- 063(*)$ &,$- 063(*)$ & 1,000 &,$- 162(* *)$ \\
$\begin{array}{l}\text { Aprendizaje } \\
\text { Rápido e }\end{array}$ &,$- 240(* *)$ &, $206(* *)$ &, $279(* *)$ &,$- 162(* *)$ & 1,000 \\
$\begin{array}{l}\text { Inaplicable } \\
\text { N }\end{array}$ & 522 & 522 & 522 & 522 & 522 \\
\hline \\
$\begin{array}{l}* * \text { La correlación es significativa al nivel de } 0.01 \\
\text { *. La correlación es significativa al nivel de } 0.05\end{array}$
\end{tabular}

Al aplicar el test de Kruskal Wallis se corroboró la relación entre el rendimiento en matemática y las dimensiones de creencias sobre esta ciencia. Como se muestra en la Tabla 4, los alumnos y alumnas ubicados en el nivel bajo creen más en la presencia de una habilidad que se requiere desde el momento del nacimiento para aprender la Matemática, lo que los aleja más de desarrollar una actitud en la que implique esfuerzo personal para aprender dicha ciencia, de la misma forma dichos estudiantes consideraron que el conocimiento matemático posee una estructura simple, radicada en la autoridad, el aprendizaje de esta ciencia ocurre a la primera vez y difícilmente tenga aplicación en la vida. Los alumnos y alumnas con alto aprovechamiento académico obtuvieron valores más bajos en este sentido, sin embargo, mostraron los valores más altos en cuanto a la relatividad del conocimiento matemático, es decir, cuando poseen una creencia de mayor apertura a la estabilidad, concibiendo su carácter tentativo y flexible alcanzan resultados docentes más altos. Dicho resultado corrobora el análisis realizado a partir de las correlaciones que se muestran en la Tabla 3.

Tabla 4. Test de Kruskal Wallis al Relacionar Creencias sobre la Matemática y Rendimiento Académico

\begin{tabular}{cccc}
\hline & Rangos & & \\
\hline & NivelRend & N & Rangos Medios \\
\hline \multirow{3}{*}{ Habilidad Innata } & Bajo & 249 & 278.58 \\
& Medio & 171 & 263.51 \\
& Alto & 98 & 204.01 \\
& Total & 518 & \\
Conocimiento Simple & Bajo & 249 & 297.53 \\
Autoridad & Medio & 171 & 241.51 \\
& Alto & 98 & 194.27 \\
& Total & 518 & \\
Conocimiento Relativo & Bajo & 249 & 230.26 \\
& Medio & 171 & 283.88 \\
& Alto & 98 & 291.24 \\
Aprendizaje Rápido & Total & 518 & \\
Inaplicable & Bajo & 249 & 305.38 \\
& Medio & 171 & 227.42 \\
& Alto & 98 & 198.91 \\
\hline
\end{tabular}


Los resultados obtenidos con la regresión (Tabla 5) arrojaron que las dimensiones de creencias que mejor predicen el rendimiento académico son, por orden de importancia: Aprendizaje rápido e inaplicable; Conocimiento simple-dependiente de la autoridad y Conocimiento relativo. La Tabla 6 muestra el valor de la significación del modelo regresión obtenido.

Tabla 5. Modelo de Regresión Obtenido al Relacionar Creencias sobre la Matemática y Rendimiento Académico.

\begin{tabular}{|c|c|c|c|c|c|}
\hline & & \multicolumn{4}{|c|}{ Resumen del Modelo ${ }^{\mathrm{d}}$} \\
\hline Modelo & Variables & $\mathrm{R}$ & $\mathrm{R}^{2}$ & $\mathrm{R}^{2}$ Ajustada & $\begin{array}{l}\text { Std. Error de } \\
\text { Estimación }\end{array}$ \\
\hline 1 & $\begin{array}{l}\text { Aprendizaje } \\
\text { Rápido } \\
\text { Inaplicable }\end{array}$ & .317 & .101 & .099 & 5.85969 \\
\hline 2 & $\begin{array}{l}\text { Conocimiento } \\
\text { Simple } \\
\text { Autoridad }\end{array}$ & $\begin{array}{c}.347 \\
\mathrm{~b}\end{array}$ & .121 & .117 & 5.79966 \\
\hline 3 & $\begin{array}{l}\text { Conocimiento } \\
\text { Relativo }\end{array}$ & $\begin{array}{c}.372 \\
\mathrm{c}\end{array}$ & .139 & .134 & 5.74532 \\
\hline
\end{tabular}

a. Predictor: (Constante), Aprendizaje Rapido Inaplicable

b. Predictor: (Constante), Aprendizaje Rapido Inaplicable, Conocimiento Simple Autoridad c. Predictor: (Constante), Aprendizaje Rapido Inaplicable, Conocimiento Simple Autoridad, Conocimiento Relativo

d. Variable dependiente: rendimiento académico

Tabla 6. Valor de la Significación del Modelo de Regresión Obtenido.

\begin{tabular}{|c|c|c|c|c|c|c|}
\hline & & & JOVA & & & \\
\hline & & Suma of & $\mathrm{df}$ & Mean Square & $\mathrm{F}$ & Sig. \\
\hline & Regresión & 1996.289 & 1 & 1996.289 & 58.140 & $.000^{\mathrm{b}}$ \\
\hline 1 & Residual & 17854.684 & 520 & 34.336 & & \\
\hline & Total & 19850.972 & 521 & & & \\
\hline & Regresión & 2393.869 & 2 & 1196.934 & 35.585 & $.000^{\mathrm{c}}$ \\
\hline 2 & Residual & 17457.104 & 519 & 33.636 & & \\
\hline & Total & 19850.972 & 521 & & & \\
\hline & Regresión & 2752.487 & 3 & 917.496 & 27.796 & $.000^{\mathrm{d}}$ \\
\hline 3 & Residual & 17098.485 & 518 & 33.009 & & \\
\hline & Total & 19850.972 & 521 & & & \\
\hline
\end{tabular}

a. Variable dependiente: Rendimiento académico

b. Predictors: (Constant), AprendizajeRapidoInaplicable

c. Predictors: (Constant), AprendizajeRapidoInaplicable, ConocimientoSimpleAutoridad

d. Predictors: (Constant), AprendizajeRapidoInaplicable

ConocimientoSimpleAutoridad, ConocimientoRelativo 
Aun cuando los resultados muestran algunas evidencias del valor predictivo de las creencias sobre el rendimiento académico, preferimos tomarlos con mucha precaución y como referencia para estudios posteriores en los que se profundice en estas relaciones de influencia y se tengan en cuenta otras variables propias de alumnos y alumnas, de los profesores y del contexto.

En el caso de los alumnos y alumnas, podría inferirse que si la actitud que se adopta ante el estudio tiene como sustento la creencia en que se requiere de una habilidad innata para aprender la Matemática y el aprendiz se percibe como un ser que no posee tal habilidad probablemente creerá que él no es una "persona de matemática” y perderá el interés por aprenderla, no obstante, se requiere profundizar en estas relaciones y otras que no han sido objeto del presente estudio.

\section{Discusión}

El análisis de la relación entre las creencias y el rendimiento académico de los alumnos ha sido objeto de numerosos autores (Bermejo, Lago, Rodríguez, Dopico, \& Lozano, 2002; De la Yera, 2013; González, 2003; Rosario et al., 2012; Schommer, Calvert, Gariglietti, \& Bajaj, 1997; Schraw \& Sinatra, 2004; Schunk \& Ertmer, 2000). En el caso de Duell y Schommer-Aikins (2001) encontraron que cuanto más simple, manejable, cierto y dependiente de la autoridad considera el estudiante que es el conocimiento, más probabilidad hay de que simplifique la información aunque sea compleja, su actuación en los exámenes sea pobre, simplifique cualquier conclusión y opte por respuestas simples allí donde sea necesaria una mayor elaboración de las mismas.

De manera similar, Garofalo (1989); Kloosterman y Cougan (1994) en sus estudios con alumnos de secundaria encontraron que cuando los estudiantes creían que casi todos los problemas de matemática pueden ser solucionados por la aplicación de hechos, reglas y fórmulas tuvieron tendencia a acercarse a las tareas matemáticas de manera mecánica o confiando en la memorización.

Como la literatura profesional señala (Kardash \& Howell, 2000; Schommer, Calvert, Gariglietti, \& Bajaj, 1997), y ha sido corroborado en el presente estudio, las creencias epistemológicas de alumnos y alumnas tienen un efecto importante en el rendimiento académico, por lo que sería importante profundizar en ellas para mejorar los resultados docentes. Una alternativa puede ser provocar la reflexión sobre las habilidades que se requiere para el aprendizaje de la Matemática, las cuales no están determinadas desde el nacimiento, sino que se desarrollan a través de la participación en las distintas actividades de aprendizaje.

También podría estimularse la reflexión relacionada con que el aprendizaje no es algo que ocurre de manera rápida casi “providencial”, sino que se adquiere lentamente a través de múltiples exposiciones a la información, a la práctica continua y distribuida de las habilidades necesarias, de la reflexión acerca de los procedimientos y los procesos mentales requeridos para la solución de problemas.

Una posible línea directriz puede ser guiar a los alumnos y alumnas al análisis de su propio proceso de aprendizaje para que reconozcan que este no ocurre solamente porque el profesor exponga información, ni por la lectura del libro de texto, sino que depende 
principalmente de los esfuerzos que ellos hagan por comprender el tema, relacionarlo con sus conocimientos previos y su experiencia del mundo, pues la Matemática en mucho representa la realidad en maneras abstractas y simbólicas.

En relación a la docencia, esta puede ser enfocada de manera que los alumnos y las alumnas desarrollen una perspectiva de la Matemática como ciencia en la que se integran conceptos, habilidades y procedimientos que les permiten resolver problemas de la vida cotidiana a diferencia de verla únicamente como procedimientos para realizar en clase y obtener una calificación. Esto puede ser un elemento que provoque sinergia y contribuya a una dinámica de retroalimentación entre los logros académicos y las creencias epistemológicas.

Como han señalado Schank y Cleary (1995), si se quiere que los alumnos y las alumnas tengan la motivación por adquirir conocimiento, este debe estar vinculado a los asuntos reales de la vida. Por ello, es necesario fomentar en ellos la comprensión de que la Matemática tiene aplicación en la vida cotidiana, así se fomentará su interés por el aprendizaje. Es importante entonces contribuir al desarrollo de la perspectiva de que, aunque una parte del conocimiento es fija, otra porción significativa es en parte transitoria y debatible y que, en el caso de la Matemática, hay distintas maneras de resolver los problemas y de modelar los aspectos numéricos de la realidad.

En conclusión, se constató la relación entre las creencias epistemológicas sobre la Matemática y el rendimiento académico. Cuando los alumnos y las alumnas creen en la simplicidad del conocimiento matemático; en la autoridad como fuente en la cual radica el conocimiento; que el esfuerzo personal es una pérdida de tiempo disminuye su rendimiento académico. Por otra parte, cuando adoptan posturas epistemológicas más flexibles y relativas en relación al conocimiento matemático alcanzan mejores resultados docentes.

\section{Referencias}

Abrate, R., Pochulu, M., \& Vargas, J. (2006). Errores y Dificultades en Matemática. Análisis de causas y sugerencias de trabajo (U. N. d. V. María Ed.). Buenos Aires, Argentina: DOCUPRINT S.A.

Balbi, A. (2010). Dificultades de aprendizaje del cálculo: Contribuciones al diagnóstico psicopedagógico. Ciencias Psicológicas, 4(1).

Barberá, E., \& Gómez, C. (1996). Las estrategias de enseñanza y evaluación en matemáticas. In C. Moreno \& I. Solé (Eds.), El asesoramiento psicopedagógico: una perspectiva profesional y constructivista (pp. 383-404). Madrid: Alianza.

Bermejo, V., Lago, M. O., Rodríguez, P., Dopico, C., \& Lozano, M. J. (2002). PEI: Un programa de intervención para la mejora del rendimiento matemático. Madrid: Editorial Complutense.

Bishop, A. J. (1999). Enculturación matemática. La educación matemática desde una perspectiva cultural. Barcelona: Paidós.

Campanario, J. M., \& Otero, J. (2000). Más allá de las ideas previas como dificultades de aprendizaje: las pautas de pensamiento, las concepciones epistemológicas y las estrategias metacognitivas de los alumnos de ciencias. Enseñanza de las Ciencias, 18(2), 
155-169.

Cano, F. (2005). Epistemological beliefs and approaches to learning: their change through secondary school and their influence on academic performance. British Journal of Educational Psychology, 75, 203-221.

Carrillo, B. (2009). Dificultades en el aprendizaje matemático. Innovación y Experiencias Educativas, (16), 1-10.

Coll, C., Palacios, J., \& Marchesi, A. (2008). Desarrollo Psicológico y Educación 2. Psicología de la educación escolar (Vol. 2). España: Alianza.

Chevallard, Y., Bosch, M., \& Gascón, J. (1997). Estudiar matemáticas. Barcelona: Horsori/ICE UB.

Davis-Kean, P. E. (2005). The infl uence of parent education and family income on child achievement: The indirect role of parental expectations and the home environment. Journal of Family Psychology, 19, 294-304.

De la Yera, A. (2013). Creencias epistemológicas, Motivación hacia el Aprendizaje y Rendimiento Académico en estudiantes de Psicología. (Trabajo de Diploma), Universidad de La Habana, La Habana.

Duell, O., \& Schommer-Aikins, M. (2001). Measures of People's Beliefs About Knowledge and Learning. Educational Psychology Review, 13(4).

Edwards, A. R., Esmonde, I., \& Wagner, J. F. (2011). Learning Mathematics. New York, NY: Routledge.

Font, V. (2011). Competencias profesionales en la formación inicial de profesores de matemáticas de secundaria. Unión, 26.

González, R. (2003). Diferencias de género en el desempeño matemático en estudiantes de secundaria. http://redalyc.uaemex.mx/pdf/405/40515206.pdf

Hofer, B. (2008). Personal Epistemology and Culture. In M. S. Khine (Ed.), Knowing, Knowledge ande Beliefs. Epistemological Studies across Diverse Cultures. Australia: Springer.

INECSE. (2010). Evaluación de la educación secundaria obligatoria: datos básicos. In MEC (Ed.). Madrid.

Kloosterman, P., \& Cougan, M. C. (1994). Students' beliefs about learning school mathematics. The Elementary School Journal, 94(4), 375-388.

Kloosterman, P., \& Stage, F. K. (1992). Measuring beliefs about mathematical problem solving. School Science and Mathematics, 92(3), 109-115.

Leal, F. (2011). Epistemología personal: Depende de cómo se mire. In J. Catalán (Ed.), Psicología Educacional. Proponiendo rumbos, Problemáticas y Aportaciones (pp. 147185). La Serena, Chile: Editorial Universidad de La Serena.

Mason, L. (2003). High school students' beliefs about maths, mathematical problem solving, and their achievement in maths: A cross-sectional study. Educational Psychology, 23(1), 73-85.

MEC. (2013). Programa para la Evaluación Internacional de Alumnos de la OCDE. España: Secretaría General Técnica Subdirección General de Información y Publicaciones.

Miñano, P., \& Castejón, J. L. (2011). Variables cognitivas y motivacionales en el rendimiento 
académico en Lengua y Matemáticas: un modelo estructural. Revista de Psicodidáctica, $16,203-230$.

Morell, M. (2011). Creencias Epistemológicas, Estrategias Metacognitivas y Rendimiento Académico en estudiantes de primer año de Ingeniería. (Tesis de Maestría), Universidad de La Habana, Ciudad de La Habana.

Muis, K. R. (2004). Personal epistemology and mathematics: A critical review and synthesis of research. Review of Educational Research, 74(3), 317-377.

OCDE. (2010). Aprender para el mundo de mañana. Madrid: Santillana.

PISA. (2012). Programa para la Evaluación Internacional de los Alumnos. Informe español (Vol. I: Resultados y contexto). Madrid: Instituto Nacional de Evaluación Educativa.

Plant, E. A., Ericsson, K. A., Hill, L., \& Asberg, K. (2005). Why study time does not predict grade point average across college students: Implications of deliberate practice for academic performance. Contemporary Educational Psychology, 30, 96-116.

Reynó de los Ríos, M. L. (2014). Creencias Epistemológicas en estudiantes de Biología, Matemática y Psicología. (Tesis de Diploma), Universidad de La Habana.

Rodríguez, L. (2005). Análisis de las creencias epistemológicas, concepciones y enfoques de aprendizaje de los futuros profesores. (Tesis Doctoral), Universidad de Granada, España.

Rosário, P., Lourenço, A., Paiva, O., Núnez, J., González-Pienda, J. A., \& Valle, A. (2012). Autoeficacia y utilidad percibida como condiciones necesarias para un aprendizaje académico autorregulado. Anales de Psicología, 28(1), 37-44.

Rosario, P., Lourenço, A., Paiva, O., Rodríguez, A., Valle, A., \& Tuero-Herrero, E. (2012). Predicción del rendimiento en matemáticas: efecto de variables personales, socioeducativas y del contexto escolar. Psicothema, 24(2), 289-295.

Schank, R., \& Cleary, C. (1995). Engines for education. Nueva Jersey: Lawrence Erlbaum Associates.

Schoenfeld, A. H. (1989). Explorations of student's mathematical beliefs and behavior. Journal for Research in Mathematics Education, 20(4), 338-355.

Schommer, M. (1990). Effects of beliefs about the the nature of knowledge on comprehension. Journal of Educational Psychology, 82, 498-504.

Schommer, M. (1994). Synthesizing epistemological belief research: Tentative understandings and provocative confusions. Educational Psychology Review, 6, 293-319.

Schommer, M. (2004). Explaining the epistemological belief system: Introducing the embedded systemic model and coordinated research approach. Educational Psychologist, 39(1), 19-29.

Schommer, M., Calvert, C., Gariglietti, G., \& Bajaj, A. (1997). The development of epistemological beliefs among secondary students: A longitudinal study. Journa of Educational Psychology, 89(1), 37-40.

Schommer, M., Crouse, A., \& Rhoder, N. (1992). Epistemological beliefs and mathematical text comprehension: Believing it is simple does not make it so. Journa of Educational Psychology, 84(4), 435-443.

Schommer, M., \& Duell, O. K. (2013). Domain Specific and General Epistemological Beliefs. 
Their Effects on Mathematics. Revista de Investigación Educativa, 31(2), 317-330. http://dx.doi.org/10.6018/rie.31.2.170911

Schraw, G., \& Sinatra, G. (2004). Epistemological development and its impact on cognition in academic domains. Contemporary Educational Psychology, 29, 95-102. doi: 10.1016/j.cedpsych.2004.01.005

Schunk, D. H., \& Ertmer, P. A. (2000). Self-regulation and academic learning, self-efi cacy enhancing interventions. In M. Boekaerts, P. Pintrich \& M. Zeidner (Eds.), Handbook of self-regulation. San Diego, NY: Academic Press.

Steiner, L. (2007). The effects of personal and epistemological beliefs on performance in a college developmental mathematics class. (Tesis Doctoral), Kansas State University, Manhattan.

Vila, A., \& Callejo, M. L. (2004). Matemáticas para aprender a pensar. El papel de las creencias en la resolución de problemas. Madrid: Narcea.

Vizcaino, A., Manzano, M., \& Casas, C. (2015). Validez de constructo y confiabilidad del Cuestionario de Creencias Epistemológicas sobre la Matemática en alumnos de secundaria básica. Revista Colombiana de Psicología, 24(2), 301-316. doi: 10.15446/rcp.v24n2.43974

Walker, D. (2007). The Development and Construct Validation of the Epistemological Beliefs Survey for Mathematics. (Doctor of Philosophy), Oklahoma State University, Stillwater, Oklahoma.

Zimmerman, B. J., \& Schunk, D. (2011). Handbook of self-regulation of learning and performance.London, New York: Routledge. 